expression in vivo, although there were significant inter-patient variations. There was a significant reduction in circulating $\mathrm{T}$ cells following CMR.

Conclusion CMR was not associated with DNA damage in vivo. $\gamma-\mathrm{H} 2 \mathrm{AX}$ expression varied markedly between individuals, therefore small studies using $\gamma-\mathrm{H} 2 \mathrm{AX}$ as a marker of DNA damage should be interpreted with caution. CMR was associated with a statistically significant reduction in viable leukocytes, although the clinical relevance of the magnitude is unclear. Further work is warranted to contextualise these findings and delineate their impact.

\section{GENERATION OF A FORMULA FOR CAROTID-FEMORAL PATHLENGTH DETERMINATION FOR USE IN PWV ASSESSMENT}

Liam Brown. University of Dundee, UK

\subsection{6/heartjnl-2017-311399.10}

Background Aortic arterial stiffening is an independent predictor for future cardiovascular events. Calculation of CarotidFemoral Pulse Wave Velocity (cfPWV) is currently the clinical gold standard for measuring arteriosclerosis, with cfPWV now recommended by the European Society of Cardiology for the guidance of initiating preventative treatments. Despite this, no consensus exists on how best to obtain the pathlength component of the calculation with large intercentre variation in how this is performed. The aim of the current study was to generate a calculation to produce a standardised pathlength that can be generated from easily obtainable clinical measures.

Methods 1183 participants free from cardiovascular disease (CVD) underwent whole body MRI as part of the TASCFORCE study. The distance between the carotid and femoral vessels was obtained by tracing the arterial centreline between the two. Backward linear regression was then used to generate a formula for calculating pathlengths based on easily obtainable clinical metrics. This calculation was then validated in an external cohort of 128 individuals with and without CVD who had also undergone MRI.

Results Various allometric and cardiovascular values were included in the analysis. Carotid-femoral pathlength could be calculated as follows:

Distance $=100.36+(0.70 \times$ Age $[$ years $])+(137.89 \times$ Height $[\mathrm{m}])$ $+(0.52 \times$ Weight $[\mathrm{kg}])-(0.17 \times$ Pulse $)+(46.16[$ female], 54.32 [male]).

When compared with the actual measured distance in the original cohort this differed by $-0.05 \mathrm{~mm} \mathrm{SD}+28.5 \mathrm{~mm}$, $\mathrm{p}=0.962$ for difference. When this formula was then applied in the external validation cohort there was a small overestimation of the pathlength by $10.07 \pm 25 \mathrm{~mm}$ ( $p>0.001)$. This is comparable to clinically accepted techniques: measuring the direct distance from the carotid-femoral arteries and subtracting the measured to distance to the carotid-sternal notch, by tape measure or calliper on the body surface, results in mean overestimation of pathlength by $-23.5 \pm 38 \mathrm{~mm}$ : a value greater than that of the generated formula technique.

Conclusion Using simple allometric measures, carotid-femoral pathlength can be calculated with good accuracy. This holds promise for improving interstudy and intercentre reproducibility, thus expanding the utility and applicability of PWV calculation in clinical practice. In future, the predictive ability of the formula can be tested in disease discrimination cohorts to further assess its clinical applicability

\section{ADENOSINE STRESS T1 MAPPING: A NOVEL CONTRAST FREE METHOD TO ASSESS MYOCARDIAL PERFUSION AND ISCHAEMIA IN HYPERTROPHIC CARDIOMYOPATHY}

${ }^{1}$ Betty Raman, ${ }^{1}$ Rina Ariga, ${ }^{1}$ Masliza Mahmod, ${ }^{1}$ Stefan Piechnik, ${ }^{1}$ Alessandra Borlotti, ${ }^{3}$ Michael Jerosch-Herold, 'Jane M Francis, 'Erica Dall'Armellina, 'Sanjay Sivalokanathan, ${ }^{1}$ Vanessa Ferreira, ${ }^{2}$ Hugh Watkins, ${ }^{1}$ Stefan Neubauer. ' Oxford Centre for Clinical Magnetic Resonance Research, Division of Cardiovascular Medicine, UK; ${ }^{2}$ Radcliffe Department of Medicine, University of Oxford, UK; ${ }^{3}$ Cardiology, Brigham and Women's Hospital, UK

\subsection{6/heartjnl-2017-311399.11}

Aim The aim of this study was to assess the ability of stress T1 mapping to detect perfusion abnormalities and inducible ischaemia in hypertrophic cardiomyopathy (HCM).

Background Impaired perfusion reserve in HCM has been shown to be an independent predictor of adverse cardiovascular outcomes. CMR perfusion imaging currently requires the administration of gadolinium based contrast agents, which are contraindicated in allergy and renal failure, making non-contrast methods such as T1 mapping a safer and more affordable alternative. As adenosine stress T1 mapping has been shown to detect inducible ischaemia in patients with coronary disease, we hypothesised that stress T1 may be used to detect perfusion abnormalities in HCM with reasonable accuracy and T1 reactivity correlates with impaired myocardial blood flow (MBF) reserve and global longitudinal strain (GLS).

Method 62 subjects with no known history of coronary disease (31 controls and $31 \mathrm{HCM}$ patients) underwent CMR at $3 \mathrm{~T}$ including cine imaging, tagging, rest and stress (adenosine $140 \mathrm{mcg} / \mathrm{kg} / \mathrm{min}$ ) blood oxygen level dependent imaging (BOLD), T1 mapping (ShMOLLI), first-pass perfusion imaging and late gadolinium imaging (LGE). Rest and stress T1 values from mid ventricular slice were derived and T1 reactivity determined. ${ }^{1,2}$ MBF was estimated using Fermi function deconvolution method as previous described. ${ }^{3,4}$

Results Baseline characteristics are listed in Table 1. T1 reactivity was significantly reduced in regions of interest (ROI)

Abstract 011 Table 1 Baseline characteristics of subjects including comparison of left ventricular indices on CMR.

\begin{tabular}{|lccc|}
\hline \multicolumn{1}{c}{$\begin{array}{c}\text { Baseline } \\
\text { Characteristics }\end{array}$} & $\begin{array}{c}\text { Healthy } \\
\text { controls }\end{array}$ & HCM & P value \\
\hline Age (years) & $45 \pm 14$ & $45 \pm 13$ & 0.8 \\
\hline Male gender [no (\%)] & $71(17)$ & $74(18)$ & 0.7 \\
\hline Rest pulse, bpm & $59 \pm 10$ & $58 \pm 11$ & 0.4 \\
\hline Systolic BP & $116 \pm 10$ & $116 \pm 12$ & 0.4 \\
\hline Hypertension & 0 & 0 & \\
\hline Smoker, \%(n) & $19(6)$ & $25(8)$ & 0.54 \\
\hline Diabetes, \%(n) & $0(0 \%)$ & $0(0 \%)$ & \\
\hline Atrial fibrillation & 0 & 0 & \\
\hline NYHA functional class & 1 & $1.2 \pm 0.4$ & 0.6 \\
\hline Beta blockers & 0 & $42(13)$ & \\
\hline ARB/ACE inhibitor, $\%(n)$ & 0 & 0 & \\
\hline LV Ejection Fraction (\%) & $68 \pm 5$ & $71 \pm 13$ & 0.09 \\
\hline LVEDV Index $\mathrm{ml} / \mathrm{m}^{2}$ & $80 \pm 14$ & $84 \pm 21$ & 0.5 \\
\hline LVESV Index ml $/ \mathrm{m}^{2}$ & $59 \pm 10$ & $58 \pm 11$ & 0.4 \\
\hline Septal thickness (mm) & $9 \pm 2$ & $15 \pm 6$ & $<0.01$ \\
\hline LV mass index $\left(\mathrm{g} / \mathrm{m}^{2}\right)$ & $54 \pm 12$ & $72 \pm 28$ & 0.02 \\
\hline Presence of $\mathrm{LGE,} \mathrm{n}(\%)$ & $0(0 \%)$ & $21(67 \%)$ & \\
\hline LGE fibrosis volume(\%) & $0(0 \%)$ & $7.3 \pm 6.1$ & \\
\hline
\end{tabular}

